\title{
Sistema Open-Source de Monitoramento do Consumo de Energia Elétrica
}

\author{
Felipe A. Przysiada \\ Engenharia da Computação, Universidade Positivo \\ Curitiba, PR, Brasil \\ felipe.przysiada@up.edu.br \\ Eduardo Silva \\ Engenharia da Computação, Universidade Positivo \\ Curitiba, PR, Brasil \\ eduardo.silva@up.edu.br
}

\author{
Diego P. Merks \\ Engenharia da Computação, Universidade Positivo \\ Curitiba, PR, Brasil \\ diego.merks@up.edu.br \\ Alessandro Brawerman \\ Engenharia da Computação, Universidade Positivo \\ Curitiba, PR, Brasil \\ brawerman@up.edu.br
}

\begin{abstract}
The cost of electricity in Brazilian homes is increasingly high. This project consists of bringing a complete and easily accessible solution aiming to benefit the economy, in a much broader way, both for the end user and for the electricity generating system, which today has difficulty in meeting demand, as well as it provides a reduction in the environmental impact caused by the constant expansion of hydroelectric plants and other sources of energy. The use of this system, the Electricity Consumption Monitoring System, allows the user to have control of each equipment installed in the premises. The equipment in monitored by a device designed and built in this project. From these monitoring devices, which perform periodic measurements, it is possible to make a daily, weekly or monthly survey of the consumption of each equipment in the residence, sending alert messages, for excessive energy consumption, thus defining a user profile and even creating limitations for monthly spending. With this, the user will have the necessary resources to manage their energy consumption over the days, without having surprises at the end of the month.
\end{abstract}

\section{KEYWORDS}

Open-Source, Monitoramento, Consumo de Energia, Gerenciamento.

\section{INTRODUÇÃO}

Economizar nem sempre é uma tarefa fácil, principalmente quando se trata de uma despesa de necessidade básica, como é o caso de energia elétrica, mas quando não se pode deixar de usá-la a saída é o consumo consciente. Com base no anúncio da Agência Nacional de Energia Elétrica (ANEEL) de janeiro de 2015, o ano começou mais caro para o setor energético pois o custo da energia estava em seus patamares mais alto, considerando que as tarifas estavam mais caras em todas as regiões do país por conta das bandeiras tarifárias em vigor. Em outubro de 2019, segundo a ANEEL, os patamares de preços ainda não estavam totalmente normalizados, pois ao longo dos anos, as bandeiras tarifarias aplicadas a cada mês foram sendo alteradas.

O consumo médio do brasileiro é de $163 \mathrm{kWh}$ por residência, segundo [7], e a tarifa média do consumidor residencial, de acordo com a Aneel, é de R\$ 0,40 por kWh. Em julho de 2015, a tarifa paranaense é de $\mathrm{R} \$ 0,49$ por $\mathrm{kWh}$, chegando a aproximadamente $\mathrm{R} \$ 0,76$ por $\mathrm{kWh}$ depois de incluídos os impostos vigentes. Assim, uma conta de um consumidor residencial custa em média $\mathrm{R} \$ 123,80$ com tributos, o que pode representar boa parte da renda mensal familiar. Portanto, deve-se atentar aos equipamentos responsáveis pelos maiores consumos de energia dentro das residências e os seus modos de uso para que se possa gerenciar seus custos [2].

\subsection{Objetivos}

O uso do Sistema de Monitoramento do Consumo de Energia Elétrica, permite ao usuário ter controle de cada equipamento ao qual for instalado o dispositivo de monitoramento, desenvolvido nesse projeto. A partir desses dispositivos, que realizam medições periódicas, é possível fazer um levantamento diário, semanal ou mensal do consumo de cada equipamento da residência, e calcular o seu valor correspondente na conta de luz. Com isso, o usuário tem recursos para gerir o seu consumo de energia ao longo dos dias, sem ter surpresas no final do mês.

Esse projeto consiste em trazer uma solução completa que visa um benefício à economia, de uma forma muito mais ampla, tanto ao usuário final quanto ao sistema gerador de energia elétrica, que hoje tem dificuldade para atender à demanda, bem como propicia uma redução no impacto ambiental causado pelas constantes ampliações de hidrelétricas e outras fontes de energia.

O Sistema de Monitoramento do Consumo de Energia Elétrica é uma solução que permite ao usuário fazer a gestão do seu consumo de energia de forma dinâmica, com mensagens de alertas, para um consumo excessivo de energia, definindo um perfil de usuário e até mesmo criando limitações para o gasto mensal. Os dados obtidos por cada dispositivo de monitoramento serão transmitidos por uma rede sem fio $\mathrm{Wi}-\mathrm{Fi}$ e demonstrados em uma interface gráfica desenvolvida na plataforma Android, na forma de um aplicativo para o celular, como pode ser visto na Figura 1. 


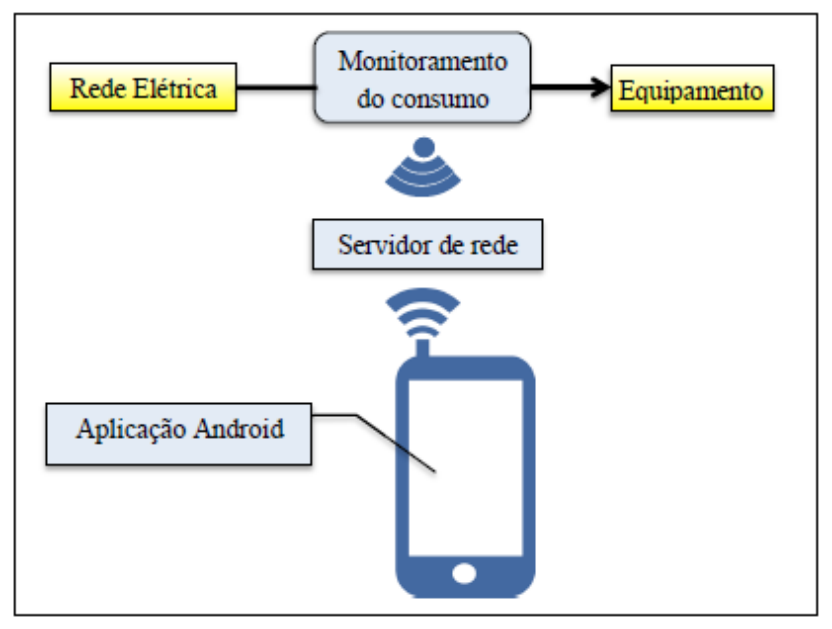

Figura 1: Diagrama da comunicação entre os elementos do sistema de monitoramento.

\section{Fundamentação Teórica}

Geralmente são dois os tipos de corrente elétrica que estão disponíveis para os consumidores residenciais. Um deles é a corrente contínua (CC), cujo fluxo de cargas elétricas, corrente elétrica, não varia nem em intensidade, nem em direção com o passar do tempo. O outro é a corrente alternada (CA), em geral senoidal, cujo fluxo de cargas varia continuamente em intensidade e sentido com o tempo. No Brasil a energia elétrica é distribuída em $110 \mathrm{~V}$ ou $220 \mathrm{~V}$ e é utilizada a frequência em $60 \mathrm{~Hz}$ [1]. Além da tensão, a grandeza elétrica que está associada ao uso da energia doméstica é a potência. $O$ termo potência, é aplicado para indicar uma quantidade de trabalho, conversão de energia, que pode ser realizado em um determinado período de tempo; isto é, a potência é a velocidade com que um trabalho é executado [1].

Como a energia convertida é medida em joules $(\mathrm{J})$ e o tempo em segundos (s), a potência é medida em joules/segundo $(\mathrm{J} / \mathrm{s})$. A unidade elétrica de medida da potência é o watt (W), definida como sendo cada watt igual a um joule por segundo [1]. A potência dissipada por um sistema ou por um dispositivo elétrico pode ser determinada em função do produto entre os valores de corrente e de tensão como mostra a Equação 1:

$$
P=V * I
$$

Como a potência é medida em watts (ou joules por segundo) e o tempo em segundos, a unidade de energia elétrica é o wattsegundo ou joule. Entretanto, a unidade watt-segundo é uma quantidade muito pequena para a maioria dos propósitos práticos, de modo que as unidades comerciais watt-hora (Wh) e quilowatthora $(\mathrm{kWh})$ foram definidas. Observe que certa quantidade de energia, em quilowatt-hora $(\mathrm{kWh})$, é expressa pelo número que exprime essa mesma quantidade de energia em watt-hora (Wh) dividida por 1000. Para exemplificar a quantidade de energia que essa unidade de potência representa, pode-se dizer que $1 \mathrm{k}$ Wh é a quantidade de energia dissipada por uma lâmpada de $100 \mathrm{~W}$ que ficou ligada por um período de 10 horas [1].

\subsection{Medição de Energia Elétrica}

Durante a evolução dos sistemas elétricos, foi constante a preocupação com a medida das grandezas envolvidas. Os primeiros dispositivos concebidos para medir o consumo de energia baseava sua medição no tempo que uma lâmpada permanecia acesa e com o avanço da tecnologia tornou-se possível medições com muito mais precisão [6].

Atualmente, aproximadamente $92 \%$ dos medidores de energia utilizados são eletromecânicos, pois possuem baixo custo e alta disponibilidade. Esse medidor é constituído principalmente pelos seguintes elementos:

- Um elemento motor, que consiste em dois circuitos magnéticos, cada um com uma bobina - uma de potencial e outra de corrente - cujos campos magnéticos resultantes são proporcionais à tensão e a corrente do circuito medido;

- Um leve disco de alumínio suspenso na região do campo magnético criado pela bobina de corrente, onde são induzidas correntes parasitas, de modo a fazer com que o disco gire no seu próprio eixo. $\mathrm{O}$ número de rotações do disco é proporcional à energia consumida pela carga em certo intervalo de tempo, fornecendo a medida do consumo de energia em quilowatt-hora (KWh).

O erro máximo de um medidor eletromecânico é de aproximadamente $2 \%$ do valor indicado para a sua faixa nominal de operação, dentro do tempo de vida previsto para o seu funcionamento. Trata-se de um valor relativamente alto, considerando as perdas que podem onerar tanto o fornecedor quanto o consumidor de energia elétrica. Além disso, devido as suas características de construção, tais como o uso de equipamentos indutivos, os medidores eletromecânicos possuem algumas limitações e restrições para que o seu funcionamento seja confiável, como: interferência na operação na presença de corrente contínua, uma vez que a presença desse tipo de corrente pode causar deformações nos fluxos magnéticos produzidos; diminuição da precisão para valores muito baixos de potência (uma vez que o atrito do conjunto mecânico é alto em relação ao torque eletromagnético produzido pelo disco); e por último, os mecanismos destes instrumentos são sensíveis a vibrações e choques produzidos por elementos externos, dificultando sua aplicação em ambientes mecanicamente instáveis ou em aplicações embarcadas [5].

Outro ponto, é que para se executar a leitura da medição, é necessário ir até o local onde o medidor está instalado e ver o valor nele registrado. Um exemplo de medidor eletromecânico pode ser visto na Figura 2 [9]. 


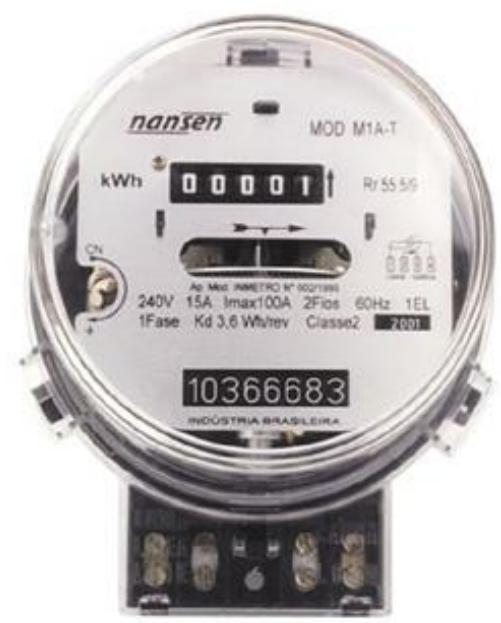

Figura 2: Medidor eletromecânico.

\subsection{Smart Meters}

Existe uma forte tendência à substituição dos medidores eletromecânicos por medidores digitais visando, além de uma maior precisão na medição, a possibilidade de leitura remota do consumo de energia ou de aplicação de uma tarifação diferenciada controlada pela faixa de horário ou pela central de distribuição.

Os smart meters, ou medidores inteligentes, são medidores eletrônicos com capacidade de processamento, armazenamento e comunicação, que vão muito além da mensuração de consumo. Eles permitem a troca de informação em tempo real e de forma bidirecional entre a empresa de eletricidade e o usuário final, e ainda o monitoramento da qualidade da energia [5].

Tecnicamente, esses equipamentos possuem precisão de $1 \%$, possibilitada principalmente pelo fato de não existirem limitações mecânicas nos elementos envolvidos no processo de medição e registro, e pela possibilidade de empregar sensores de alta precisão. Por possuírem circuitos e dispositivos baseados em semicondutores, podem ser sensíveis a surtos de tensão eventualmente presentes no sistema de distribuição. Portanto, o projeto desse tipo de dispositivo envolve necessariamente cuidados para que estes tipos de eventos não comprometam o funcionamento dos componentes sensíveis, preservando a confiabilidade e precisão [4].

Com esses dispositivos, o consumidor tem acesso a diversas informações, tais como o preço da energia fornecida e o quanto de energia já foi consumida até então ou está sendo consumida naquele momento, em $\mathrm{KW}$ ou em termos financeiros [4].

\section{Implementação}

A camada física do projeto é chamada de dispositivo de monitoramento, sendo que este tem a função de estimar o consumo de energia elétrica. A Figura 3 apresenta o diagrama em blocos que mostra uma visão geral desse elemento, que se divide em três partes principais, sendo elas:

- Medição de corrente elétrica;

- Digitalização e processamento dos dados obtidos;

- $\quad$ Transmissão sem fio Wi-Fi;

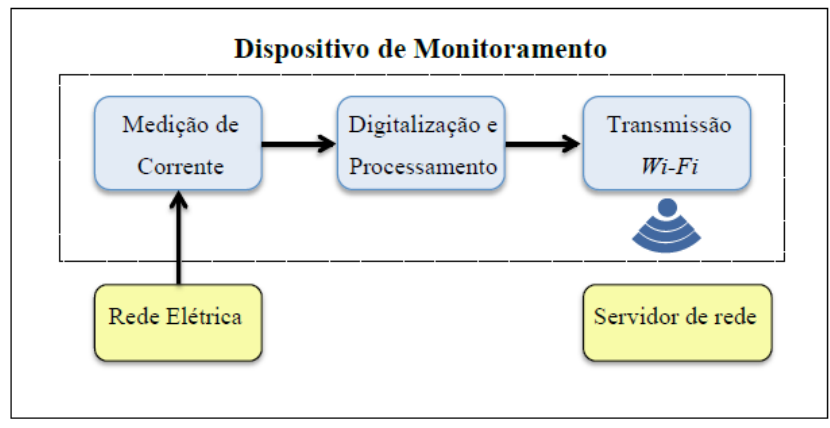

Figura 3: Diagrama em blocos das etapas do monitoramento do consumo de energia elétrica.

\subsection{Dispositivo de Monitoramento}

$\mathrm{O}$ dispositivo de monitoramento tem uma função crucial no funcionamento do sistema, pois será atribuído a ele as funções de medir o consumo instantâneo de energia, com base no sensoriamento da corrente elétrica utilizada pelo equipamento, codificar os dados obtidos utilizando um microcontrolador e transmitir a informação através de uma rede sem fio a partir de um módulo de Wi-Fi. Um exemplo de aplicação desse dispositivo pode ser visto no diagrama da Figura 4.

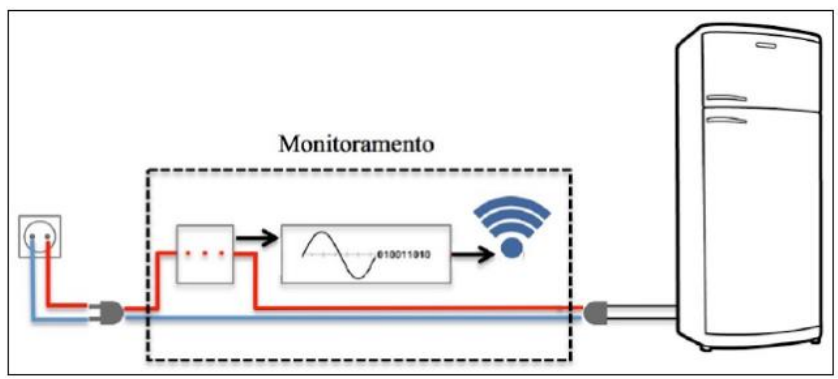

Figura 4: Exemplo de aplicação e diagrama do funcionamento do dispositivo de monitoramento: medição, digitalização e transmissão.

Para tanto foram desenvolvidas as placas de circuito impresso, considerando a melhor disposição dos componentes utilizados nas mesmas de modo a deixar prática e viável a utilização dentro do equipamento proposto. As Figuras 5, 6 e 7, apresentam o diagrama esquemático para a confecção da placa de circuito impresso, o layout do roteamento das trilhas e a placa já montada com os devidos componentes, respectivamente. Todos os circuitos que envolvem projeto da placa de circuito impresso foram desenvolvidos usando o software de desenvolvimento Eagle.

Os principais componentes a serem citados são o microcontrolador atmega, o modulo Wi-Fi ESP32 e o sensor de corrente. 


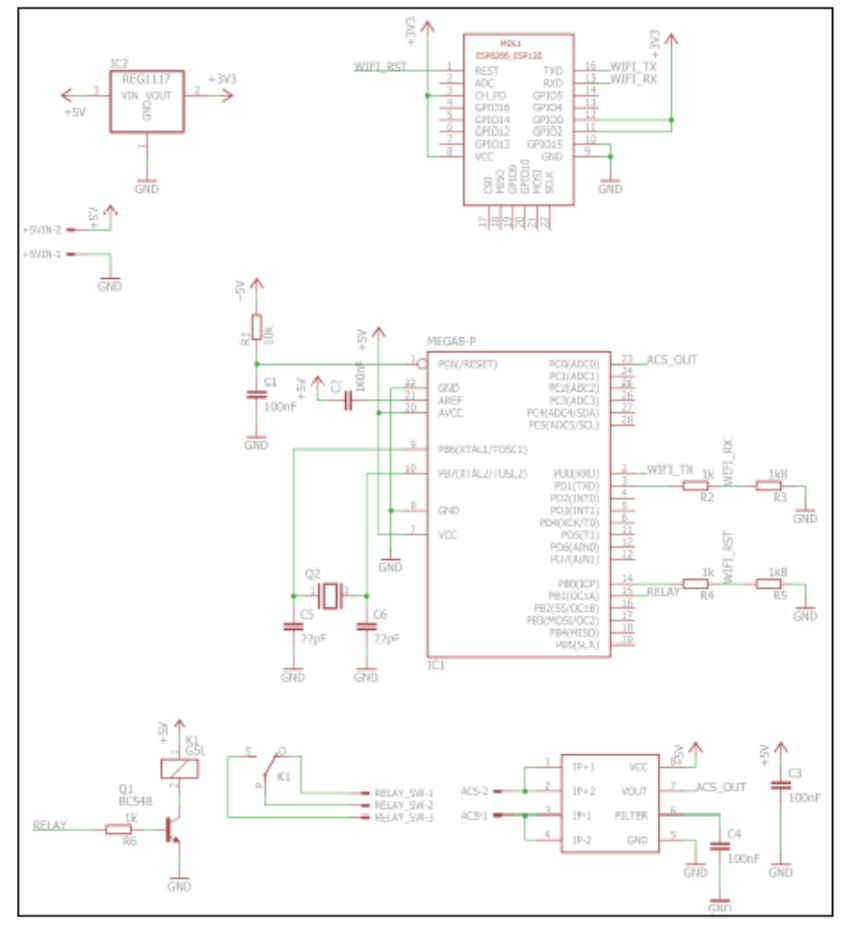

Figura 5: Diagrama esquemático do dispositivo de monitoramento.

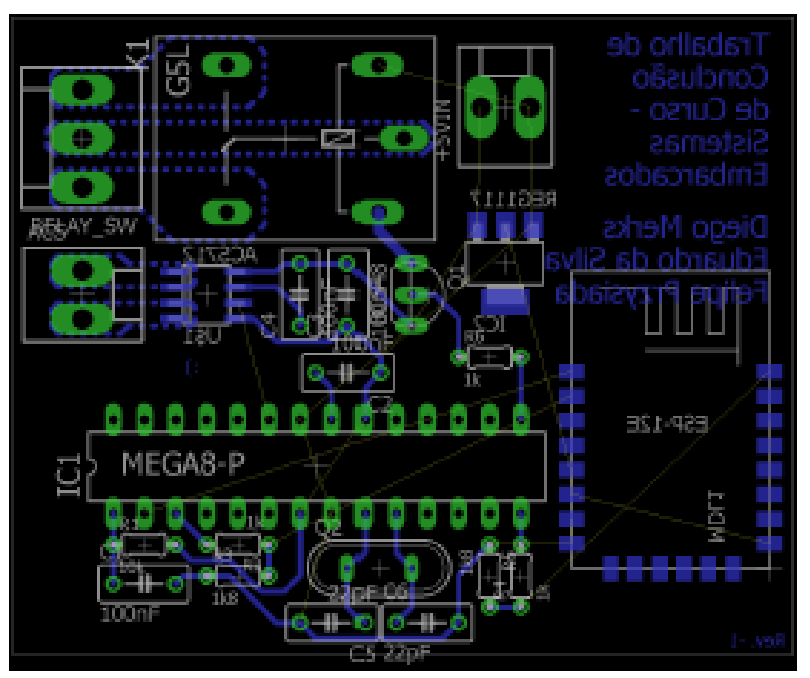

Figura 6: Roteamento da placa do dispositivo de monitoramento.

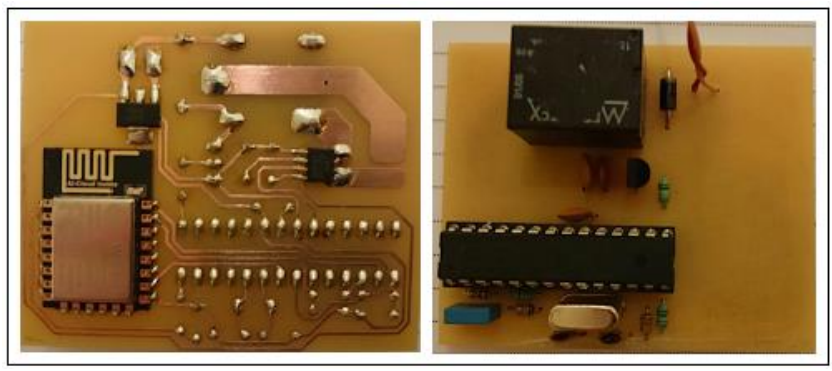

Figura 7: As duas faces da placa montada do dispositivo de monitoramento.

\subsection{Medição de Corrente}

O processo de medição de corrente é fundamental para estimar o consumo de energia elétrica. Uma vez que a tensão do equipamento será informada, em volts, pelo usuário, o consumo de energia pode ser estimado com base na corrente elétrica consumida pelo equipamento ou eletrodoméstico que estiver ligado ao medidor. O modelo utilizado nesse projeto é o ACS712 $-20 \mathrm{~A}$, que tem capacidade de realizar leituras de correntes de até 20 ampères [3].

Quando é aplicada corrente através do condutor de cobre, um campo magnético é gerado o qual é detectado pelo elemento incorporado no "elemento Hall". A intensidade do campo magnético é proporcional à intensidade da corrente através do circuito de condução, proporcionando uma relação linear entre a tensão de saída, ou tensão de Hall, e a corrente de entrada.

\subsection{Processamento Digital}

Todo o processamento digital desenvolvido para que os dados coletados dos dispositivos de monitoramento sejam interpretados, armazenados e transmitidos foram divididos em duas etapas, o "Servidor de Rede" e o "Firmaware", que estão descritos a seguir.

3.3.1 Firmaware. As duas funções básicas do firmware são, processar os dados da medição de corrente elétrica e transmiti-los para o servidor utilizando a rede Wi-Fi.

3.3.1.1 Aquisição. A aquisição dos dados é realizada, a partir do microcontrolador ATmega88, usando um conversor analógicodigital (ADC), que é capaz de receber valores analógicos de tensão, entre 0 e $5 \mathrm{~V}$, e converte-los para um valor digital com uma resolução de 10 bits.

Para o cálculo de corrente, utilizou-se da escala fornecida pelo fabricante do sensor de corrente, que determina um nível de tensão analógica, em volts, diretamente proporcional a cada valor de corrente medido pelo sensor, em ampères, como mostra a Figura 8. Como os valores de tensão obtidos correspondem aos valores de pico, ou seja, os máximos de cada medição realizada, portanto, para que sejam enviados valores com mais precisão, se faz o ajuste desses valores para uma média RMS, ou tensão eficaz. 


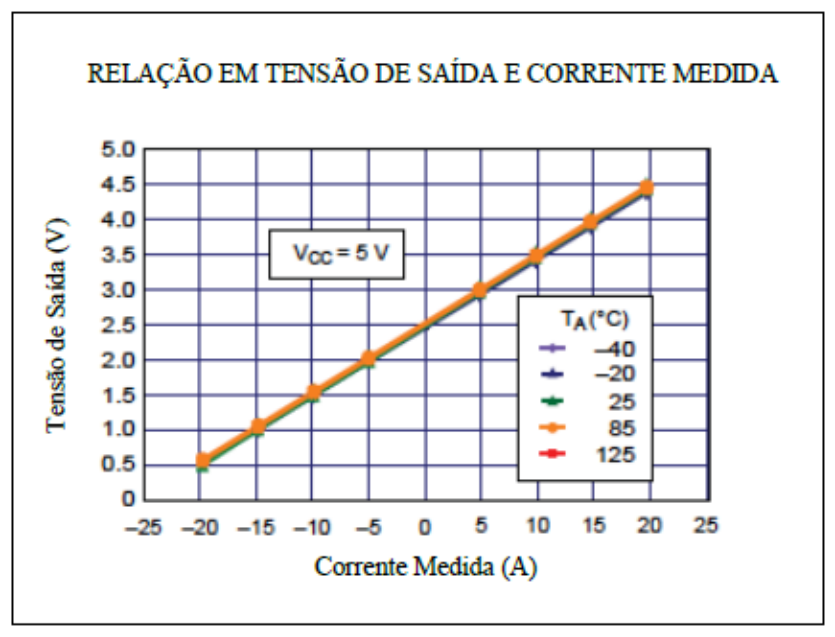

Figura 8: Relação tensão corrente do sensor.

3.3.1.2 Conexão e Transmissão. Para a conexão do dispositivo de monitoramento a uma rede Wi-Fi, é enviado um valor inteiro que pode variar de um a duzentos e cinquenta e cinco, usado como identificador de dispositivo, como por exemplo, o envio do número ' 1 ' corresponde ao "Medidor 1". A partir do momento que a conexão é realizada com êxito, o dispositivo de monitoramento, inicia transmissão de dados referentes às medições realizadas pelo sensor de corrente em forma de valores inteiros, que pode variar de zero a vinte mil. As informações recebidas pelo servidor são armazenadas no banco de dados e em seguida é aguardado um novo recebimento. Este ciclo é mantido enquanto o dispositivo de monitoramento estiver conectado à rede.

3.3.2 Servidor de Rede. O servidor de rede tem a função de interligar o dispositivo de monitoramento à aplicação Android, como pode ser vista na Figura 9, para que o usuário possa ter acesso a todas as informações de medições e controles de forma simples. Para isso, o servidor deve se conectar à rede Wi-Fi, criada pelo dispositivo de monitoramento, processar ou interpretar todos os dados enviados pelo mesmo, assim como, armazenar em banco de dados os valores medidos durante um período de até trinta dias e ainda executar os comandos, sobre os equipamentos ou eletrodomésticos, enviados pela aplicação Android.

O servidor é responsável por executar uma sequência de etapas, sendo que a primeira delas é, após a inicialização do servidor, fazer a verificação das informações no banco de dados e atualização dos alertas. Nesta etapa do processo, acontece o cálculo da potência a partir dos dados de corrente existentes no banco de dados, para todas as informações disponíveis, gerando a atualização dos valores de gasto diário, semanal e mensal. Assim que estes valores são atualizados, é feita a comparação com os valores de meta, que serão pré-definidos pelo utilizador no aplicativo, enviando uma mensagem de alerta no caso do gasto mensal se aproximar ou ultrapassar o valor de meta definida. Está função é executada a cada período de dez minutos ou quando o utilizador faz o pedido das informações sobre o consumo, via aplicativo.

É importante ressaltar que existe apenas um servidor para controlar as conexões feitas pelo dispositivo de monitoramento e pelo aplicativo, ou seja, existem fluxos distintos para cada um destes, sendo:

- Fluxo de controle para o dispositivo de monitoramento: resume-se ao recebimento das informações enviadas pelo mesmo, no qual é recebido um valor inteiro que pode variar de um a cem para identificar o dispositivo de monitoramento, além do recebimento de outro valor inteiro que pode variar de zero a vinte mil, este servindo como informação da corrente medida pelo dispositivo de monitoramento, previamente identificado. As informações recebidas são armazenadas no banco de dados e em seguida é aguardado um novo recebimento. Este ciclo é mantido enquanto o dispositivo de monitoramento estiver conectado à rede.

- Fluxo de conexão do aplicativo: gera um fluxo de controle diferenciado. Neste controle o servidor deve receber um valor inteiro igual a zero do aplicativo, para assim identifica-lo como aplicativo. Com o aplicativo identificado, o servidor envia as informações referentes a nome do equipamento, estado de ligamento, cômodo, tensão de funcionamento, meta existente, consumo diário, consumo semanal, consumo mensal e o alerta, caso este último exista. Após isso, o servidor aguarda que o aplicativo envie as alterações feitas pelo utilizador, para poder atualizar as informações do banco de dados, ou então, um valor de terminação de conexão, para indicar que o usuário não está mais utilizando o aplicativo e nenhuma alteração nos valores iniciais aconteceu.

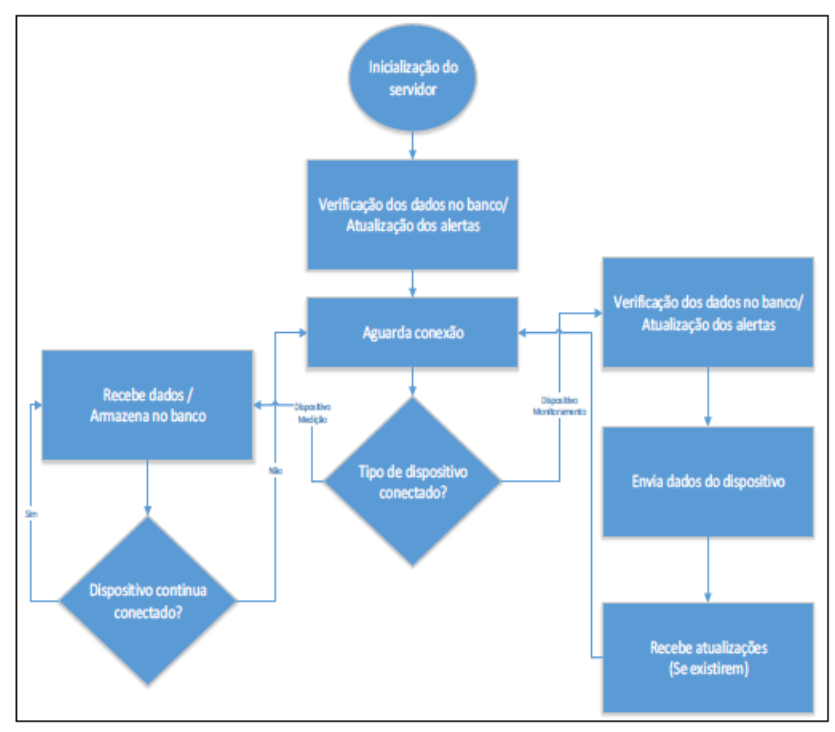

Figura 9: Fluxograma do servidor.

\subsection{Transmisão Wi-Fi}

Após o processo de aquisição dos dados, obtidos a partir da corrente elétrica medida pelo sensor, e o processamento dos mesmos, esses valores serão adaptados para a transmissão, seguindo um protocolo de comunicação, de tal forma que o servidor de rede possa recebê-los e interpretá-los como um valor digital correspondente ao valor da corrente medida. Para a transmissão foi escolhido o dispositivo ESP8266 [8]. 


\subsection{Aplicação Android}

A aplicação Android que será desenvolvida nesse projeto visa criar uma interface na qual o usuário terá acesso aos dados de consumo e também poderá controlar os equipamentos que estiverem sendo monitorados. Nesse aplicativo o usuário poderá escolher qual equipamento pretende monitorar e acompanhar o seu consumo de energia, que poderá ser consultado sempre que desejado. $\mathrm{O}$ mesmo informa o consumo instantâneo, mas também informa um total aproximado da sua conta de luz com base numa média diária, semanal ou mensal.

Também é possível pré-definir um valor de limite mensal a ser gasto e, com base nisso, em caso de consumo excessivo de um ou mais equipamentos, o usuário será informado através de mensagens de alerta.

A Figura 10, ilustra como será feita escolha dos dispositivos de monitoramento, aqui chamados de "Medidor", que se pretende monitorar. Ao escolher o "Medidor 1", por exemplo, o aplicativo apresentará os dados referentes ao consumo de energia de um eletrodoméstico qualquer ligado a ele, as informações fornecidas pelo usuário e o estado de funcionamento do equipamento, que poderá ser usado como controle do equipamento, ligando e desligando de acordo com a necessidade a partir do aplicativo, como mostrado na Figura 11.

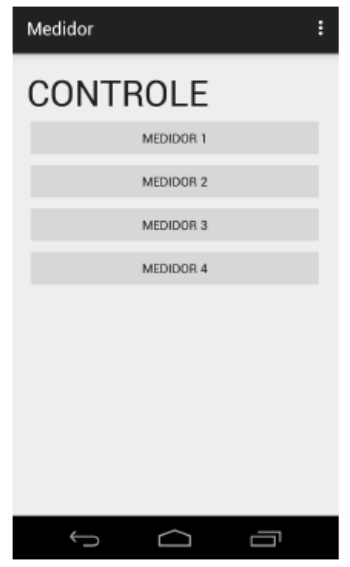

Figura 10: Tela de início do aplicativo.

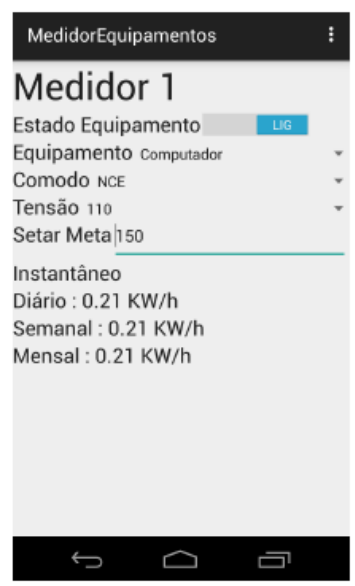

Figura 11: Informações sobre o medidor.
Após escolher o medidor que será usado, o usuário irá nomeálo e configurá-lo. O primeiro item de seleção que aparece na tela do aplicativo é o "Equipamento". Essa lista apresenta algumas opções de eletrodomésticos que, como mostra a Figura 12 (a), será usada para a escolha do nome, apenas para que o usuário saiba qual o equipamento será monitorado. O segundo item de seleção é o "Cômodo". Essa lista apresenta algumas opções de cômodos, onde estará o eletrodoméstico em questão, como mostra a Figura 12 (b).

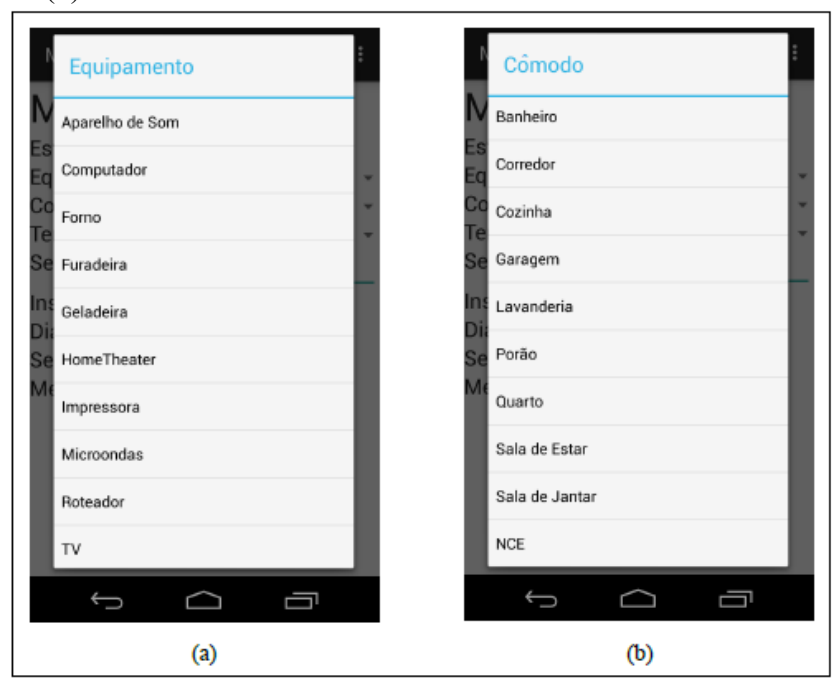

Figura 12: Configuração do sistema de medição

Por ser um projeto open-source, todos os códigos e esquemáticos desenvolvidos se encontra disponível no site: https://github.com/Przysiada/Gerenciador-de-Energia para acesso irrestrito de utilizadores e desenvolvedores.

\section{Teste e Resultados}

Desde o início do desenvolvimento desse projeto, o hardware não se mostrou um grande obstáculo, por ser um processo mais lógico do que físico, de modo que os circuitos montados e projetados atenderam totalmente a necessidades. As placas de circuito impresso foram confeccionadas com o tamanho ideal para ocupar o espaço interno do corpo do equipamento. $\mathrm{O}$ dispositivo funciona em modo Plug\&Play, ou seja, basta conectá-lo à rede de energia e sua conexão à rede de comunicação acontece de forma automática. A Figura 13, mostra que o dispositivo conta com um LED indicador de conexão, que acenderá na cor vermelha, indicando que ele está desconectado da rede ou na cor verde quando a conexão for completada com êxito. 


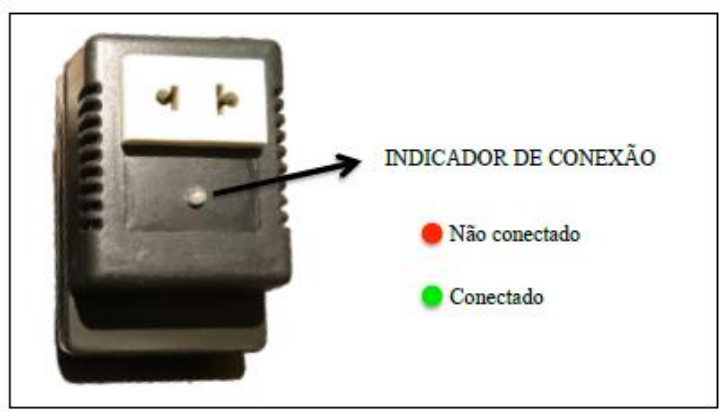

Figura 13: Indicador de conexão do dispositivo de monitoramento.

Os testes relacionados ao hardware foram realizados com o objetivo de verificar as conexões e alcance do módulo Wi-Fi em relação ao roteador. Neste teste, foi obtido $100 \%$ de êxito em um ambiente livre de obstáculos a uma distância, entre medidor e roteador, de aproximadamente 8 metros e com conexões durando em média 2 horas.

Além disso, se executaram-se testes relacionados as acurácias de medição. O processo de medições seguiu um padrão que determinava, para um período de tempo, o maior, o menor e o valor médio de corrente medido. Também foi analisado o número, total e médio, de medições realizadas nesse tempo. Com base nos valores nominais de tensão e potência, de cada equipamento, foi possível comparar os resultados obtidos com os esperados e, com base nisso, definir parâmetros para medições futuras. Na Figura 14, abaixo, estão listados os equipamentos testados e os parâmetros adotados para o padrão de medição.

\begin{tabular}{|l|c|c|c|c|c|}
\cline { 2 - 6 } \multicolumn{1}{c|}{} & $\begin{array}{c}\text { Ferro } \\
\text { de } \\
\text { solda }\end{array}$ & $\begin{array}{c}\text { Geladeira } \\
\text { (com o motor } \\
\text { desligado) }\end{array}$ & $\begin{array}{c}\text { Tela de } \\
\text { Monitor }\end{array}$ & $\begin{array}{c}\text { Notebook } \\
\text { (com a bateria } \\
\text { carregada) }\end{array}$ & $\begin{array}{c}\text { Notebook } \\
\text { (com a bateria } \\
\text { descarregada) }\end{array}$ \\
\hline Potência Nominal [W] & 150 & 52,1 & 80 & -- & 180 \\
\hline Tensão Nominal [V] & 110 & 220 & 110 & 110 & 110 \\
\hline Maior Medição [A] & 1,78 & 0,43 & 1,02 & 1,47 & 2,11 \\
\hline Menor Medição [A] & 1,31 & 0,19 & 0,64 & 1,00 & 1,59 \\
\hline Média de Corrente Medida [A] & 1,41 & 0,24 & 0,74 & 1,12 & 1,72 \\
\hline Potência Medida [W] & 155,1 & 52,8 & 81,4 & 123,2 & 189,2 \\
\hline Número de Medições Geradas & 3340 & 22565 & 45679 & 32305 & 12702 \\
\hline Número de Medições por Minuto & 162,05 & 186,49 & 204,84 & 186,73 & 181,46 \\
\hline Tempo de Cada Medição [h:mm:ss] & $0: 20: 37$ & $2: 01: 19$ & $3: 43: 55$ & $2: 53: 41$ & $1: 10: 34$ \\
\hline
\end{tabular}

Figura 14: Relação de valores obtidos, de acordo com o padrão adotado para as medições de teste.

\section{Considerações Finais e Sugestões}

De acordo com os testes realizados, foi possível mensurar a sua usabilidade, cumprindo com a proposta inicial de informar ao usuário o consumo médio de energia elétrica, de modo individual, dos equipamentos ou eletrodomésticos que se deseja monitorar.

O aplicativo Android propicia fácil navegação e interface amigável, informando de forma prática todos os dados relacionados às medições e status dos equipamentos. Uma deficiência encontrada ao longo do desenvolvimento, foi a necessidade de manter a conexão com o servidor local, reduzindo a sua mobilidade.

$\mathrm{O}$ aspecto do dispositivo de monitoramento ficou similar a uma fonte de alimentação, usada tipicamente em dispositivos eletrônicos, ou seja, uma aparência familiar de tamanho prático e leve. Por ser de fácil utilização, como um equipamento Plug\&Play, quando ligado à rede de energia, sua conexão à rede de comunicação acontece de forma automática. Seu uso não querer conhecimento técnico nem configurações. $\mathrm{O}$ equipamento conectado ao dispositivo de monitoramento, deverá apenas ser identificado no aplicativo Android pelo usuário. Para a realização de medições com valores de corrente superiores a 20 ampères, valor máximo para a qual o dispositivo foi projetado, o projeto de hardware deverá ser revisto.

A medição do consumo de energia elétrica apresentou, em média, um erro de $2,79 \%$ em relação aos valores nominais dos equipamentos, percentual bastante próximo do encontrado em medidores eletromecânicos. Desta forma, vale ressaltar, que a medição de forma precisa, não é o propósito desse projeto, e sim permitir a gestão dos gastos baseado em um consumo médio que cada equipamento monitorado obteve em um período de tempo. Com base nas informações obtidas, na fase de testes, foi possível definir uma margem de erro geral que não impacta sobre a conta de luz de forma decisiva, confirmando assim sua usabilidade. Deve-se ressaltar que as medições não servem de parâmetros para contestar valores medidos por equipamento homologado pela concessionária de energia.

Estão listadas abaixo, algumas sugestões de aprimoramentos para uma versão futura desse projeto, sendo elas:

1. Melhorar a interface gráfica do aplicativo Android;

2. Aumentar a quantidade de informações para o usuário;

3. Criar um perfil de utilização, a partir da base de dados de cada usuário;

4. Portar o servidor local para um servidor Web melhorando sua mobilidade;

5. Aprimorar cálculos de medições que reduzam os erros;

6. Realizar ensaios severos, para melhor definir os parâmetros máximos de medição.

\section{REFERENCES}

[1] Agência Nacional de Energia Elétrica (Ed. 3) 2008. Atlas de Energia Elétrica do Brasil.

[2] Agência Nacional de Energia Elétrica (ED. 1) 2019 Histórico de Acionamento das Bandeiras Tarifárias até novembro de 2019.

[3] Allegro Microsystems, Llt, ACS712 Complete Datasheet, http://www.alldatasheet.com/datasheetpdf/pdf/168326/ALLEGRO/ACS712.html

[4] Companhia Paranaense de Energia, Uso Eficiente de Energia na Sua Casa, http://www.copel.com/hpcopel/residencial/informacoes.jsp

[5] Companhia Paranaense De Energia, Simulador de Consumo de Energia Elétrica, http://www.copel.com/hpcopel/simulador/index.htm

[6] David Dahle (2012) Samuel Gardiner's lamp hour meter

[7] Empresa de Pesquisa Energética (Ed. 1) 2019. Anuário Estatístico de Energia Elétrica 2018.

[8] Espressif Systems, ESP8266 Complete Datasheet, https://www.adafruit.com/images/productfiles/2471/0AESP8266_Datasheet_EN_v4.3.pdf

[9] Nansen, Medidores Eletromecânicos, http://www.nansen.com.br 